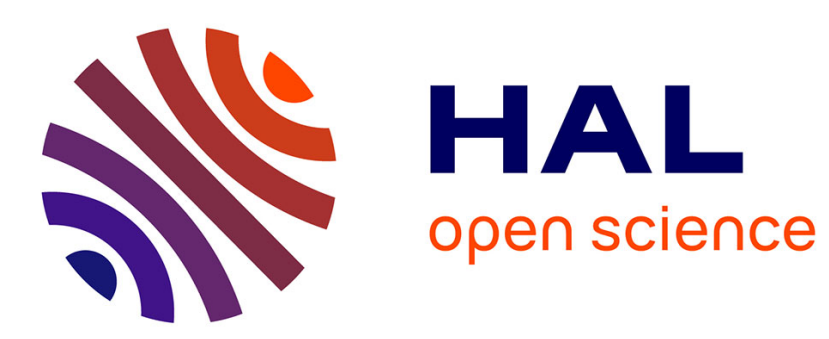

\title{
Unscreened polyelectrolyte chain in d dimensions : a Monte Carlo simulation
}

\author{
A. Baumgärtner
}

\section{To cite this version:}

A. Baumgärtner. Unscreened polyelectrolyte chain in d dimensions : a Monte Carlo simulation. Journal de Physique Lettres, 1984, 45 (11), pp.515-521. 10.1051/jphyslet:019840045011051500 . jpa00232376

\section{HAL Id: jpa-00232376 https://hal.science/jpa-00232376}

Submitted on 1 Jan 1984

HAL is a multi-disciplinary open access archive for the deposit and dissemination of scientific research documents, whether they are published or not. The documents may come from teaching and research institutions in France or abroad, or from public or private research centers.
L'archive ouverte pluridisciplinaire HAL, est destinée au dépôt et à la diffusion de documents scientifiques de niveau recherche, publiés ou non, émanant des établissements d'enseignement et de recherche français ou étrangers, des laboratoires publics ou privés. 
Classification

Physics Abstracts

36.20E -05.40

\title{
Unscreened polyelectrolyte chain in $d$ dimensions : a Monte Carlo simulation
}

\section{A. Baumgärtner}

Institut für Festkörperforschung der Kernforschungsanlage Jülich, Postfach 1913, D-5170 Jülich, F.R.G.

(Reçu le 12 mars 1984, accepté le 16 avril 1984)

\begin{abstract}
Résumé. - L'exposant $v$ décrivant les propriétés des configurations d'une chaîne isolée de polyélectrolyte est déterminé par une méthode de Monte Carlo en dimension $3 \leqslant d \leqslant 7$. Des chaînes consistant en, au plus, 320 liens rigides librement attachés sont examinées. Nous trouvons un comportement de la distance entre extrémités de la chaîne $R \sim N^{v}$ avec $v=3 / d$, en accord avec les calculs auto-cohérents et les théories de type Flory. Il diffère de l'exposant donné par les théories Lagrangiennes à zéro composante et le groupe de renormalisation dans l'espace réel qui conduisent à $v=2 /(d-2)$ pour $4<d \leqslant 6$.
\end{abstract}

Abstract. - The exponent $v$ describing the configurational properties of an isolated polyelectrolyte chain in dimensions $3 \leqslant d \leqslant 7$ is determined using Monte Carlo methods. Freely jointed chains consisting of up to $N=320$ rigid links are considered. We find an end-to-end distance $R \sim N^{v}$ with $v=3 / d$ which is in agreement with self-consistent calculations and Flory type theories. It differs from zero component Lagrangian theory and real space renormalization group calculations which give $v=2 /(d-2)$ for $4<d \leqslant 6$.

\section{Introduction.}

Linear polyelectrolytes exhibit a quite different behaviour than neutral polymers [1] because of the long range Coulomb interactions between monomers. Recently the conformation properties of an isolated unscreened polyelectrolyte chain for dimensionalities $d \geqslant 3$ have been investigated using renormalization group methods [2-5]. The main result was that the exponent $v$ of the end-to-end distance $R \propto l N^{v}(l=$ size of the unit, $N=$ number of units in the chain) is given for $4<d \leqslant 6$ by [2-4]

$$
v_{\mathrm{RG}}=2 /(d-2) .
$$

This result has been obtained from zero component Lagrangian theory [2-4] and from a special type of real space renormalization group calculation (" decimation along the chemical sequence of a polymer chain ") $[2,6]$. The result (1) is in contrast to self-consistent calculations and Flory type theories [7] for the exponent $v$ which give

$$
v_{\mathrm{F}}=3 / d
$$


for $3 \leqslant d \leqslant 6$. This simple result is obtained crudely by minimizing the free energy consisting of the elastic energy and the bare Coulomb energy of an unscreened polyelectrolyte chain [2, 7].

However, it has been pointed out in reference [5] that corrections in the field-theoretic model are important below an unspecified marginal dimension $d^{*}<6$ where the unphysical exact result $v_{R G}(1)$ is expected to break down. Hence, the hitherto existing renormalization group treatments of unscreened polyelectrolyte chains should be reexamined critically.

In this paper we presented Monte Carlo results for end-to-end distance, radius of gyration, internal energy and internal distances of an isolated unscreened polyelectrolyte chain in $3 \leqslant d \leqslant 7$ dimensions. Our results exhibit an exponent $v=v_{\mathbf{F}}$.

\section{Model and simulation technique.}

As a model we consider the freely jointed chain consisting of $N$ beads which are connected by $N-1$ rigid links of length $l=1$. The beads interact pairwise according to a generalized Coulomb potential in $d$ dimensions [2]

$$
U_{i j}=\frac{e^{2}}{\Omega_{d} r_{i j}^{d-2}}
$$

where $r_{i j}$ is the distance between beads $i$ and $j . \Omega_{d}=2 \pi^{d / 2} / \Gamma\left(\frac{1}{2} d\right)$ is the area of a $d$ dimensional hypersphere of radius unity.

Configurations of the chain have been generated using the " slithering-snake " technique $[8,9]$ : starting from an arbitrary configuration, one first selects one of the ends of the chain at random and then removes this end link of the chain and adds it to the other end, specifying randomly the orientation of the link. The new state is accepted as a new one if the transition probability $W$ exceeds a random number $0<\eta<1$, otherwise it is rejected and the old configuration is retained and is treated as if a move has been made. The transition probability is constructed so that it satisfies detailed balance with the equilibrium distribution $P_{0} \propto \exp \left(-E / k_{\mathrm{B}} T\right)$, where $E=\sum_{i, j} U_{i j}$ is the total energy of the chain : if $\Delta E=E_{\text {old }}-E_{\text {new }} \geqslant 0, W=1$; if $\Delta E<0$ then $W=\exp \left(\Delta E / k_{\mathrm{B}} T\right)$. In all simulations the temperature was given by $k_{\mathrm{B}} T=10 e^{2} / \Omega_{d} l^{d-2}$.

Chain conformations thus generated are characterized by three quantities : the mean square end-to-end distance $\left\langle R^{2}\right\rangle$, the mean square radius of gyration

$$
\left\langle S^{2}\right\rangle=\sum_{i=1}^{N-1} \sum_{j=i+1}^{N}\left\langle r_{i j}^{2}\right\rangle / N^{2}
$$

and the total energy of the chain

$$
\langle\tilde{E}\rangle=\sum_{i=1}^{N-1} \sum_{j=i+1}^{N}\left\langle r_{i j}^{2-d}\right\rangle .
$$

Average values have been estimated from about $1000 \mathrm{~N}^{2}$ Monte Carlo cycles (MCC) for $N=320$, $2000 N^{2} \mathrm{MCC}$ for $N=160,4000 N^{2} \mathrm{MCC}$ for $N=80$, and $8000 N^{2} \mathrm{MCC}$ for $N=40$ and $N=20$. Initial relaxation effects have been safely excluded in the averaging. All simulations have been performed on the IBM 3081 and partially on the CRAY X-MP. On the CRAY X-MP the program ran about 40 times faster than on the IBM 3081. 


\section{Results and discussions.}

The estimated values of $\left\langle R^{2}\right\rangle$ and $\left\langle S^{2}\right\rangle$ are summarized in tables I and II, and plotted on a $\log \log$ scale in figures 1 and 2 respectively. Figure 1 and figure 2 clearly demonstrate that $\left\langle R^{2}\right\rangle / N^{2} l^{2} \propto N^{2 v-2}$ and $\left\langle S^{2}\right\rangle / N l^{2} \propto N^{2 v-1}$ with $v=v_{\mathrm{F}}=3 / d$, for $3 \leqslant d \leqslant 6$ and $v=1 / 2$ for $d=7$, which is in agreement with self-consistent calculations and Flory type theories. For comparison, the broken lines in figures 1,2 indicate the expected power law behaviour for $d=5$ according to renormalization group calculations $[2-4,6]$ with $v=v_{\mathrm{RG}}=2 /(d-2)$. Of course, we cannot exclude a possible crossover to a different behaviour for very long chains

Table I. - Normalized mean squared end-to-end distance $\left\langle R^{2}\right\rangle / N^{2} l^{2}$ for dimensions $3 \leqslant d \leqslant 7$. The numbers in brackets denote the estimated error in the last digit of the preceding number.

\begin{tabular}{|c|l|l|l|l|c|}
$N$ & 20 & 40 & 80 & 160 & 320 \\
\hline$d=3$ & $0.102(6)$ & $0.096(6)$ & $0.104(6)$ & $0.104(8)$ & - \\
4 & $0.086(2)$ & $0.0565(8)$ & $0.0412(8)$ & $0.0300(4)$ & $0.023(2)$ \\
5 & $0.074(1)$ & $0.0410(9)$ & $0.0240(6)$ & $0.0138(4)$ & $0.0082(2)$ \\
6 & $0.068(2)$ & $0.0345(9)$ & $0.0179(9)$ & $0.0094(1)$ & - \\
7 & $0.062(2)$ & $0.031(1)$ & $0.015(1)$ & $0.0075(2)$ & -
\end{tabular}

Table II. - Normalized squared radius of gyration $\left\langle S^{2}\right\rangle / N l^{2}$ for dimensions $3 \leqslant d \leqslant 7$.

\begin{tabular}{|c|c|c|c|c|c|}
$N$ & 20 & 40 & 80 & 160 & 320 \\
\hline$d=3$ & $0.286(5)$ & $0.505(8)$ & $1.02(2)$ & $1.96(8)$ & - \\
4 & $0.250(4)$ & $0.326(6)$ & $0.466(6)$ & $0.702(8)$ & $1.03(9)$ \\
5 & $0.221(4)$ & $0.247(6)$ & $0.295(6)$ & $0.348(8)$ & $0.40(2)$ \\
6 & $0.21(1)$ & $0.216(8)$ & $0.230(8)$ & $0.245(8)$ & - \\
7 & $0.192(2)$ & $0.195(2)$ & $0.197(3)$ & $0.198(3)$ & -
\end{tabular}

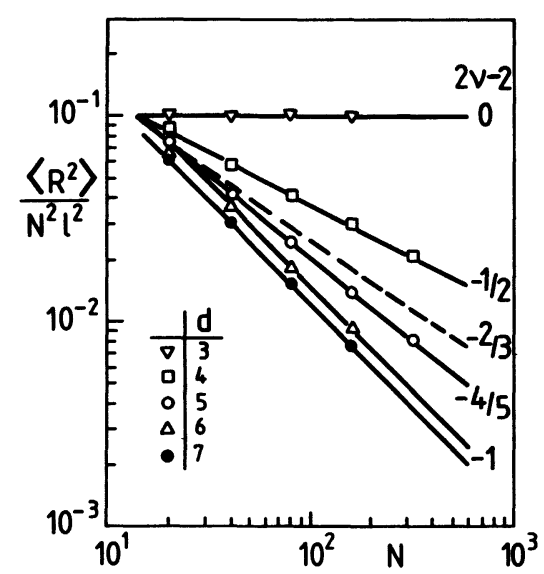

Fig. 1. - Log-log plot of normalized mean square end-to-end distances $\left\langle R^{2}\right\rangle / N^{2} l^{2}$ versus $N$ for dimensions $3 \leqslant d \leqslant 7$. The resulting exponents $2 v-2$ are given at the right hand side of the figure. 
$(N \gg 320)$; but on the other hand already for $N \leqslant 160$ our results are in full agreement with the non-controversial predictions for $d=3$ and $d \geqslant 6$; i.e. $v=1$ and $v=1 / 2$ respectively, which support the reliability of our results for $d=4$ and $d=5$.

Even more convincing evidence for $v=v_{\mathrm{F}}$ is provided by the examination of average internal distances $\bar{r}_{i j} \equiv\left\langle r_{i j}^{2}\right\rangle^{1 / 2}$ presented in figures 3 and 4 for $d=3$ and $d=4$ respectively. A log-log

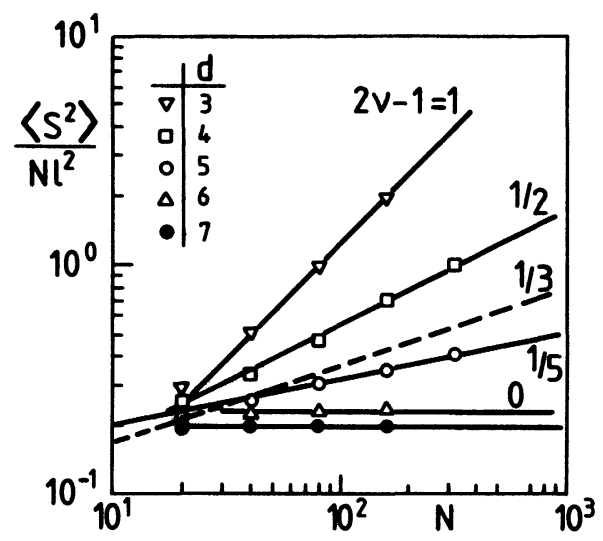

Fig. 2. - Log-log plot of normalized mean square radius of gyration $\left\langle S^{2}\right\rangle / N l^{2}$ versus $N$ for dimensions $3 \leqslant d \leqslant 7$. The resulting exponents $2 v-1$ are shown at the right hand side of the figure.

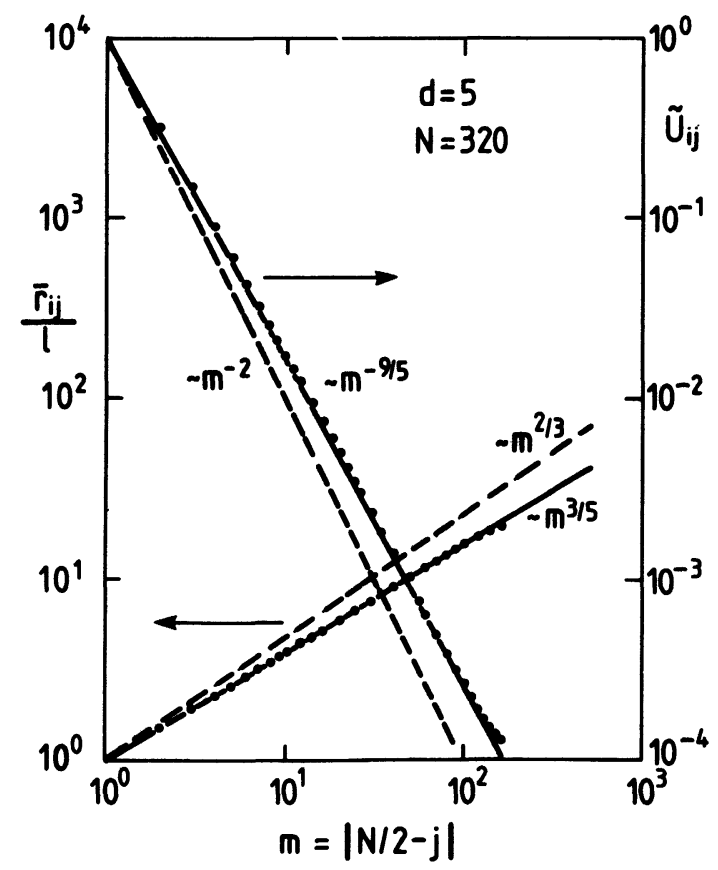

Fig. 3. - Log-log plot of average internal distances $\bar{r}_{i j} \equiv\left\langle r_{i j}^{2}\right\rangle^{1 / 2}$ and Coulomb energy $\tilde{U}_{i j}=\left\langle r_{i j}^{2-d}\right\rangle$ between beads $i=N / 2$ and $N / 2+1 \leqslant j \leqslant N$ for $N=320$ and $d=5$ versus chemical distance $m=$ $|N / 2-j|$. For comparison, the broken lines correspond to a power law with $v=v_{\mathrm{RG}}=2 / 3$. 


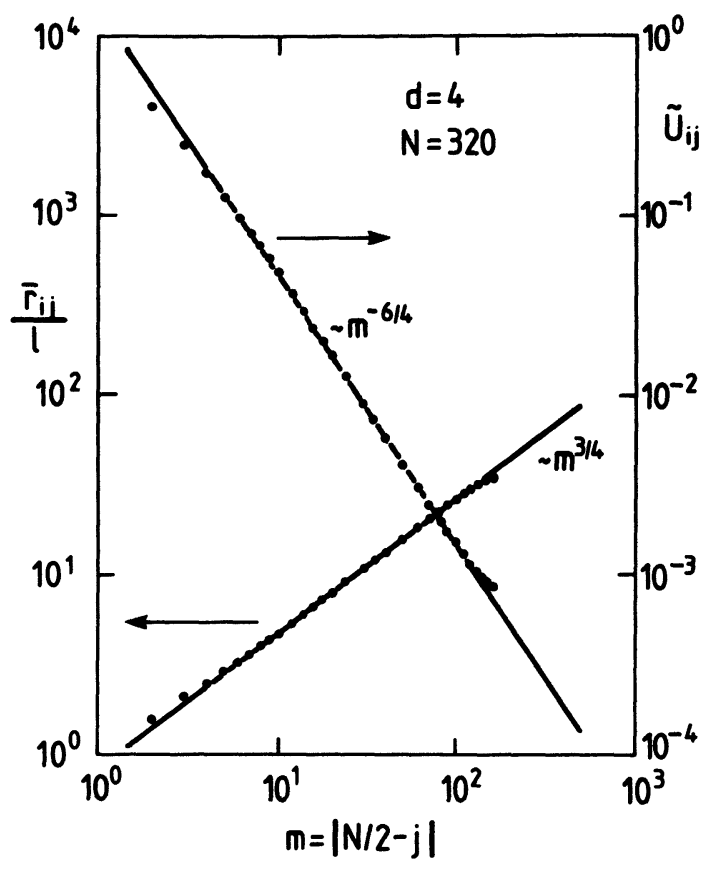

Fig. 4. - Compare figure 3, but now for dimension $d=4$.

plot of $\bar{r}_{i j} / l$ versus $|i-j|$ with fixed $i=N / 2$ and $N / 2+1 \leqslant j \leqslant N$ for $N=320$ exhibit clearly the behaviour

$$
\bar{r}_{i j} / l \propto|i-j|^{v}, \quad v=3 / d
$$

and correspondingly the average Coulomb energy between the beads $i$ and $j$ (neglecting short distances correlations and end effects)

$$
\tilde{U}_{i j}=\left\langle r_{i j}^{2-d}\right\rangle=A|i-j|^{v(2-d)}, \quad v=3 / d .
$$

The broken lines in figure 3 indicate the power laws according to $v=v_{\mathbf{R G}}$. The initial deviation of $\bar{r}_{i j}$ from its asymptotic power law (6) which is observed in figure 4 , is related to short range correlations.

The total Coulomb energy of the chain as a function of $N$ and $3 \leqslant d \leqslant 6$ is shown in figure 5 in a semi-log plot. For $4 \leqslant d \leqslant 6$ the energy $\langle\tilde{E}\rangle / N l^{2-d}$ approach a final value, which is crudely estimated by using equation (5), (7) and $A \approx 1$, which give

$$
\lim _{N \rightarrow \infty}\langle\tilde{E}\rangle / N l^{2-d}=\zeta(3(d-2) / d), \quad 4 \leqslant d \leqslant 6
$$

where $\zeta(x)$ is the Riemann zeta function. The behaviour of $\langle\tilde{E}\rangle / N l^{2-d}$ for finite $N$ according to (7) is indicated in figure 5 by the full lines. The actual asymptotic values obtained by Monte Carlo calculations deviate from (8) by factors of $1.47,1.38$ and 1.21 for $d=4,5$ and 6 respectively, which reflect the corrections of short range correlations.

In three dimensions the total Coulomb energy per bead diverge logarithmically [7]. Using (5) and (7), we obtain for $N \rightarrow \infty$

$$
\langle\tilde{E}\rangle / N l \propto \ln N, \quad d=3 .
$$




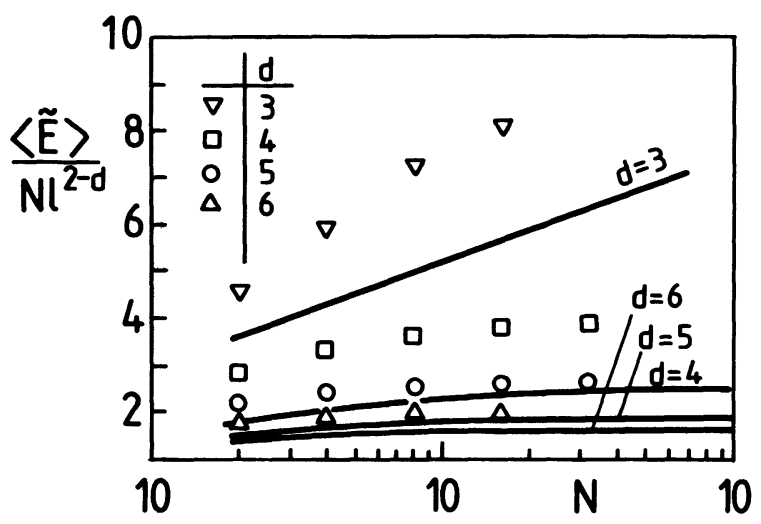

Fig. 5. - Semi-log plot of average total Coulomb energy $\langle\tilde{E}\rangle / N l^{2-d}$ [Eq. (5)] versus $N$ for $3 \leqslant d \leqslant 6$. The full lines have been calculated using equations (5), (7) with $A=1$.

Table III. - Normalized energy $\langle\tilde{E}\rangle / N l^{2-d}$ for dimensions $3 \leqslant d \leqslant 7$.

\begin{tabular}{|c|c|c|c|c|c|}
$N$ & 20 & 40 & 80 & 160 & 320 \\
\hline$d=3$ & $4.54(4)$ & $5.94(4)$ & $7.23(6)$ & $8.2(4)$ & - \\
4 & $2.83(3)$ & $3.33(3)$ & $3.62(3)$ & $3.79(3)$ & $3.86(3)$ \\
5 & $2.14(2)$ & $2.40(2)$ & $2.52(2)$ & $2.60(2)$ & $2.65(2)$ \\
6 & $1.78(2)$ & $1.94(2)$ & $2.00(2)$ & $2.03(2)$ & - \\
7 & $1.55(2)$ & $1.63(2)$ & $1.67(2)$ & $1.69(2)$ & -
\end{tabular}

The actual values of $\langle\tilde{E}\rangle / N l$ for finite $N$ assuming $A \approx 1$ are calculated using (7). This is shown in figure 5 by the full line labelled by $d=3$. The deviations from our Monte Carlo results are probably due to short range correlations and finite size effects. Further investigation for $d=3$ at several temperatures might be useful in order to study the internal correlations as a function of coupling parameter $\lambda$ defined by $k_{\mathrm{B}} T=\lambda e^{2} / 4 \pi l$, and chain length $N$.

\section{Summary and conclusions.}

In the present work we presented the results of Monte Carlo simulations of single unscreened polyelectrolyte chains in three to seven dimensions. From the estimated values of end-to-end distance $\left\langle R^{2}\right\rangle$, radius of gyration $\left\langle S^{2}\right\rangle$ and internal correlations $\bar{r}_{i j}$ for various chain lengths $20 \leqslant N \leqslant 320$, we found that the correlation length exponent $v$ is in agreement with self-consistent calculations and Flory-type theories [7] $v_{\mathrm{F}}=3 / d$, but in disagreement with renormalization group calculations $[2-4,6] v_{\mathrm{RG}}=2 /(d-2)$ valid for $4<d \leqslant 6$.

Our results seem to support recent suggestions on the modification of $v_{\mathrm{RG}}$ below a marginal dimensionality $d^{*}<d_{\mathrm{c}}=6$, which was concluded from the appearance of relevant higher order corrections in the renormalization theory of $n=0$ model Lagrangian [5].

Reasons why de Gennes' real space renormalization group method, which has been successfully applied to uncharged polymer chains [6], leads to results which are different from the present Monte Carlo calculations are quite unclear. This point is being currently investigated. 


\section{References}

[1] Oosawa, F., Polyelectrolytes (M. Dekker, New York) 1971.

[2] Pfeuty, P., Velasco, R. M., De Gennes, P. G., J. Physique Lett. 38 (1977) L-5.

[3] Pfeuty, P., J. Physique Colloq. 39 (1978) C2-149.

[4] Jug, G., Rickayzen, G., J. Phys. A 14 (1981) 1357.

[5] Jug, G., J. Physique Lett. 42 (1981) L-409.

[6] De Gennes, P. G., Scaling Concepts in Polymer Physics, Chap. XI (Cornell University Press, Ithaca, N. Y.) 1979.

[7] De Gennes, P. G., Pincus, P., Velasco, R. M., Brochard, F., J. Physique 37 (1976) 1461.

[8] Kron, A. K., Polymer Sci. USSR 7 (1965) 1361 ;

Kron, A. K., Ptitsyn, O. B., Polymer Sci. USSR 9 (1967) 847.

[9] Wall, F. T., Mandel, F., J. Chem. Phys. 63 (1975) 4592. 